\title{
Oscillations in a half-empty bottle
}

\author{
Andréane Bourges, Amélie Chardac, Aude Caussarieu, Nicolas Plihon, and Nicolas Taberlet* \\ Univ Lyon, Ens de Lyon, Univ Claude Bernard, CNRS, \\ Laboratoire de Physique, Département de Physique, F-69342 Lyon, France
}

(Dated: December 13, 2019)

\begin{abstract}
When a half-empty bottle of water is pushed to roll on a flat surface, the oscillations of the fluid inside the bottle induce an overall jerky motion. These velocity fluctuations of the bottle are studied through simple laboratory experiments accessible to undergraduate students and can help them grasp fundamental concepts in mechanics and hydrodynamics. We first demonstrate through an astute experiment that the rotation of the fluid and the bottle are decoupled. The equations of motion are then derived using a mechanical approach while the hydrodynamics of the fluid motion is explained. Finally the theory is tested against two benchmark experiments.
\end{abstract}

\section{INTRODUCTION}

\section{The phenomenon}

When a half-empty bottle initially resting horizontally on a flat table is pushed, the fluid it contains is set into motion, which leads to an oscillatory rolling motion of the bottle. Figure 1 shows an example of the trajectory of a $1 \mathrm{~L}$ glass bottle half-filled with water, manually pushed at the time indicated by the dashed line. The space-time diagram showing the position of the cap as a function of time clearly illustrates the intermittent overall motion of the bottle. In the particular case shown in Figure 1, the bottle undergoes a stop-and-go motion with a typical frequency of a few Hertz. The goal of the present paper is to understand and model this oscillatory motion.

The phenomenon of surface gravity waves on water and oscillations of fluid has been studied for a wide variety of container shapes ${ }^{1,2}$ and the dependence of frequency on curvature in a vertical cylinder is known ${ }^{3}$. However, when the container is subjected to an external force and free to move, the problem becomes more complex and the free surface of the fluid adopts different shapes, depending on the excitation and the geometry of the container. This issue is of great importance in a wide range of applications involving liquid transport, from the problems encountered by space agencies in aerospace vehicles ${ }^{4,5}$, to tank carriages on highway or rail roads ${ }^{6,7}$.

The motion of a soft-drink can on an incline was studied by Jackson et al. ${ }^{8}$. The authors used water (and varied the filling fraction of the can) as well as granular matter (lead shots and glass marbles) and proposed a model which describes the limiting cases of non-viscous and infinitely viscous fluids. Later, $\operatorname{Lin}^{9}$ compared the rolling dynamics of cans fully filled with liquid water and solid ice, Ireson and Twidle ${ }^{10}$ showed that shaking a can of soda noticeably affects its rolling speed and Micklavzina $^{11}$ investigated the influence of the fluid viscosity.

In this article the bottles are modeled by simpler cylindrical tubes. The main goal of this article is to study the oscillating speed of a half-empty bottle. The ratio of filling is therefore fixed to one half and the fluid used in all of our experiments is tap water.
What students can learn from this problem

The oscillatory motion of a bottle is a simple hands-on experiment which can illustrate important concepts in solid and fluid mechanics. The Euler-Lagrange equations $^{12}$ are used to investigate the motion of a coupled system, and the conservation of energy during an elastic shock allows one to predict the bounce of a bottle on a wall. Moreover, the phenomenon exposes hydrodynamics concepts to explain a fluid motion: characteristic times relevant to the phenomenon are identified, the validity of a potential flow approach ${ }^{13}$ is discussed and the eigenmodes of fluid motion in a container are studied.
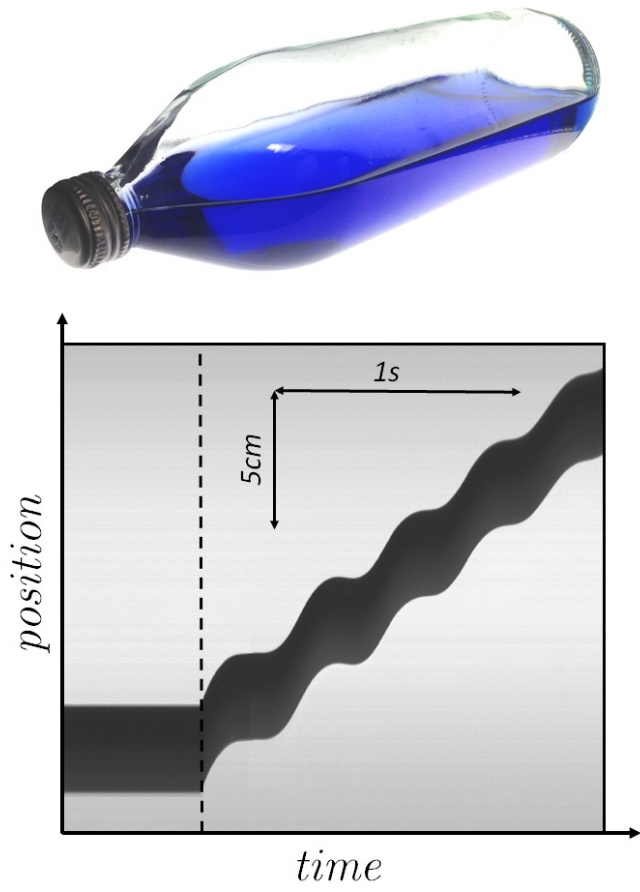

FIG. 1. Top: picture of a half-empty glass bottle. Bottom: space-time plot showing the motion of the cap after the bottle was given a push. The oscillations in speed, adding to the overall forward motion at constant velocity, are clearly visible. Diagram obtained from a 1500-pixel line video at 500 FPS. 


\section{Outline of the paper}

In order to model the overall oscillating motion of a half-empty cylinder, we will first focus in part II on the coupling between the water and the solid and show that their rotation are decoupled for short times. The motion of the fluid within a half-empty still cylinder, known as sloshing, is presented in part III, and allows one to define the moment of inertia of the water, which can be used in the equations of motion derived from the purely mechanical model developed in section IV. Finally, part V will show experimental results of oscillations obtained when a bottle bounces on a wall or rolls down a steep slope.

\section{ROTATIONAL COUPLING}

\section{A. Race down a slope}

The study of a bottle rolling down an incline ${ }^{8-11}$ is extremely informative regarding the motion of the fluid inside the rolling container. Jackson et al. ${ }^{8}$ in particular, showed that the velocity of an empty can is noticeably less than that of a can filled with water. This indicates that the relative moment of inertia of an empty can (i.e. normalized by mass $\times$ radius ${ }^{2}$ ) must be larger than that of a full or half-empty can. Indeed, although the fluid clearly contributes to the overall mass of the system (and therefore its weight), it may not contribute significantly to the total moment of inertia since, in general, it is not in solid-body rotation within the container.

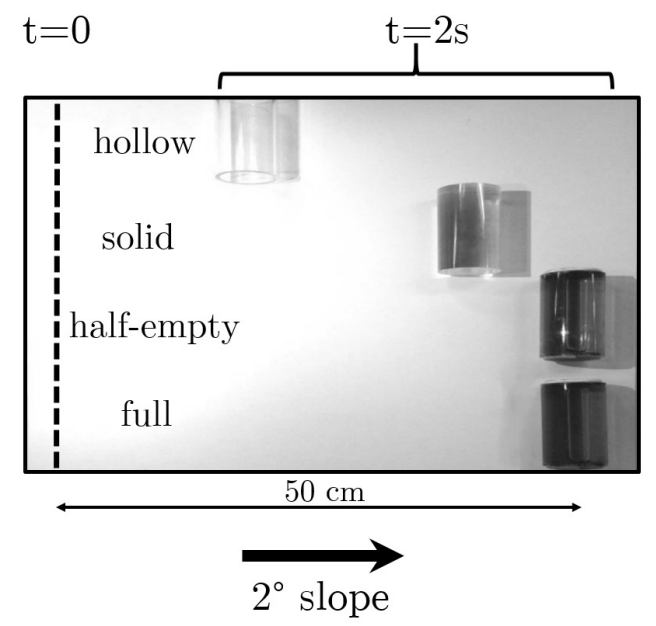

FIG. 2. Picture showing four bottles racing down a $2^{\circ}$ slope. The initial positions are shown by the dashed line and the picture is taken after $2 \mathrm{~s}$ of rolling. The race shows that the moments of inertia of half-empty and full bottles are lower than that of the solid cylinder, which indicates that the water undergoes little rotation but only a simple translation.

We have reproduced the experiments by releasing four 'bottles' of identical radius $(3 \mathrm{~cm})$ and length $(12 \mathrm{~cm})$ on a $2^{\circ}$ slope: a hollow tube (or empty bottle), a solid Plexiglass cylinder, a half-empty bottle and a full bottle.
Note that on this gentle slope, all objects roll with no slip. A picture taken $2 \mathrm{~s}$ after the start of the race is shown in figure 2 while figure 3 displays the positions in time of the various bottles, measured from particle tracking (using the 'Analyze Particle' tool of ImageJ, a free software developed by NIH, on a $1500 \times 1000$ pixels video at 30 FPS).

As expected, the hollow tube is visibly slower than the solid cylinder. Figure 3 shows that the velocity increases roughly linearly in time, which indicates that friction plays no role. The behavior of the half-empty and full bottles is remarkable: both roll down the slope at greater speeds than the hollow tube and the empty bottle, which indicates a smaller moment of inertia. Their motion is close to that of a simple friction-free sliding block (indicated by a dashed line in figure 3). Again, these results indicate that the water inside the bottle (whether halfempty or full) undergoes little rotation but is instead simply translated. In conclusion, this simple experiment shows that the rotation of the bottle is decoupled from that of the fluid (over the short duration of the race).

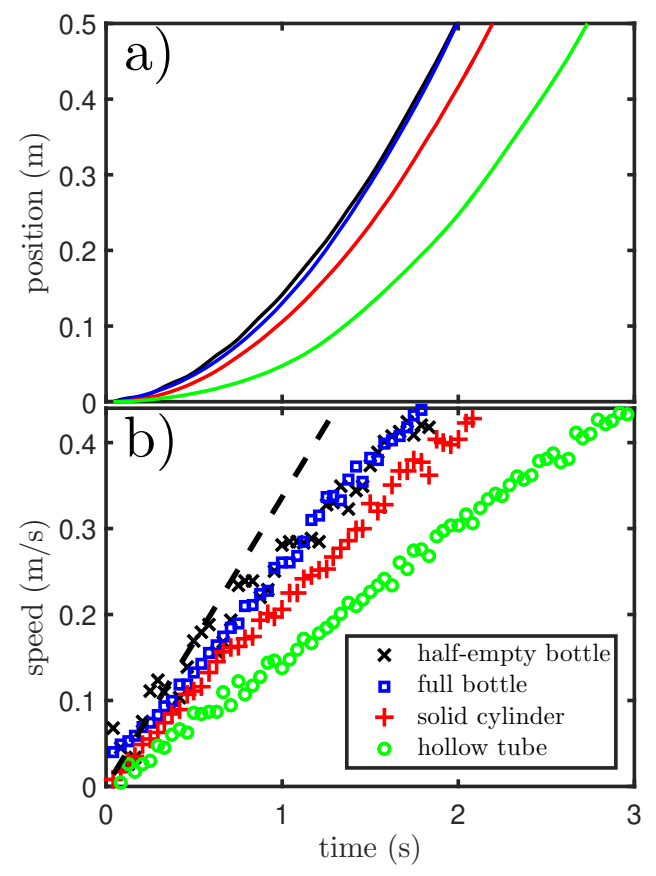

FIG. 3. Top: position along the $2^{\circ}$ slope for an empty, halfempty and full bottles and a solid cylinder. Bottom: velocity computed as the derivative of the position. The full and halfempty bottles are the fastest whereas the empty bottle is the slowest. The greater acceleration of the half-empty and full bottles is due to their reduced moment of inertia. The dashed line indicates a constant acceleration: $g \sin 2^{\circ}$. 


\section{B. Diffusion time}

Results from the previous paragraph indicate that a bottle can spin without inducing rotation within the water. This only holds for short times, less than the typical diffusion time of momentum. Indeed, over longer times the viscosity of the fluid should induce motion of the fluid. In this paragraph we discuss the momentum diffusion in a vertical rotating bottle. We have performed a simple experiment in which a tall cylinder (of height $400 \mathrm{~mm}$ and radius $R=50 \mathrm{~mm}$ ) is placed on a rotating table (an old record-player). The motion of the water is followed using neutrally buoyant tracers (700 $\mu \mathrm{m}$ polystyrene beads) from a $1000 \times 1000$-pixel video recorded at $30 \mathrm{FPS}$. At time $t=0$, the rotation is started (at 33 RPM).

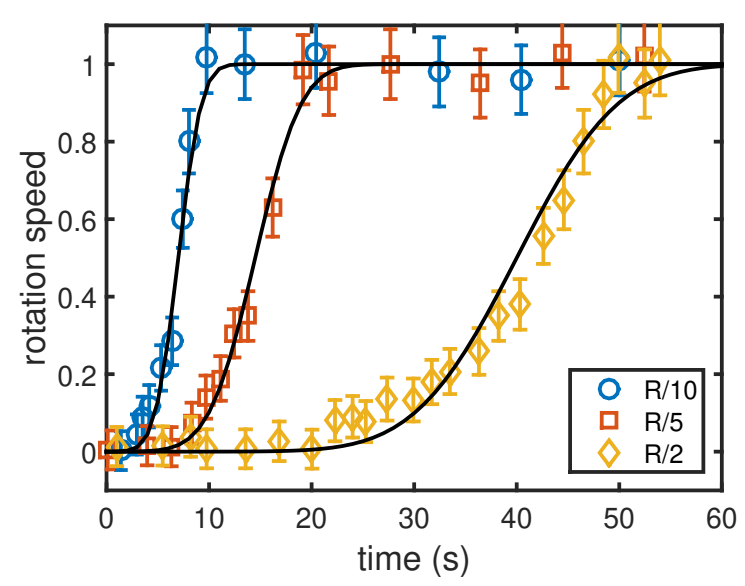

FIG. 4. Rotation speed of water (measured at $R / 10, R / 5$ and $R / 2$ from the wall) in a tall vertical cylinder initially at rest at set into rotation at $t=0$. The lines are guides for the eye.

Figure 4 shows the time evolution of the local rotation speed of the water (normalized by that of the rotating support) measured at various distances from the outer wall $(R / 10=5 \mathrm{~mm}, R / 5=10 \mathrm{~mm}$ and $R / 2=25 \mathrm{~mm})$. It takes about $30 \mathrm{~s}$ for the fluid at $R / 2$ (i.e. half-way from the wall to the center) to start rotating and $50 \mathrm{~s}$ to reach a steady velocity. Even more interestingly, it takes typically 10 s for the fluid near the bottle wall (at $R / 10$ ) to be set into motion. Therefore, one can conclude that over short times (typically a few seconds) the vast majority of the fluid is not affected by the rotation of the bottle.

An estimate of the diffusion length of the velocity within the fluid (or its momentum), $\delta$, over the duration, $T$, of a typical experiment can be given knowing the kinematic viscosity, $\nu$, of the fluid ${ }^{14,15}: \delta=\sqrt{\nu T} \simeq 3 \mathrm{~mm}$ for $T=10 \mathrm{~s}$, which is compatible with the experimental data of figure 4 and negligible compared to the radius of the bottle. For an oscillatory motion of typical frequency $1 / T=3 \mathrm{~Hz}$ (see figure 1 ), the boundary layer is given by penetration length ${ }^{14,15}$ (analogous to the electromag- netic skin-depth $\left.{ }^{16-18}\right): \delta=\sqrt{\nu T / \pi} \simeq 0.3 \mathrm{~mm}$, which is an order of magnitude smaller.

In conclusion, we have shown that the rotation of the fluid and of the bottle are decoupled as long as the experiment lasts typically for less than $10 \mathrm{~s}$.

\section{SLOSH DYNAMICS IN A STILL BOTTLE}

In this section the motion of the fluid in a still bottle is discussed. This slosh dynamics was studied as early as the XIX $^{\text {th }}$ century by Rayleigh ${ }^{19}$ and a comprehensive review can be found in $\mathrm{Lamb}^{20}$ and Ibrahim ${ }^{21}$.

\section{A. Potential flow}

In a horizontal cylinder the first eigenmode of sloshing displays a flat (although not constantly horizontal) free surface which oscillates up and down. Rayleigh ${ }^{19}$ showed that under the assumption of a potential flow (where in particular the viscosity of the fluid can be neglected) the velocity field can be written with separated variables, i.e. as a product of a function of time and a second function of the position. This allows to define the moment of inertia for the rotation of the fluid around the center of the bottle:

$$
I_{s}=\pi \rho R^{4} L\left(\frac{4}{\pi^{2}}-\frac{1}{4}\right) \simeq 0.31 m R^{2},
$$

where $\rho$ is the density of the fluid and $L$ the length of the bottle.
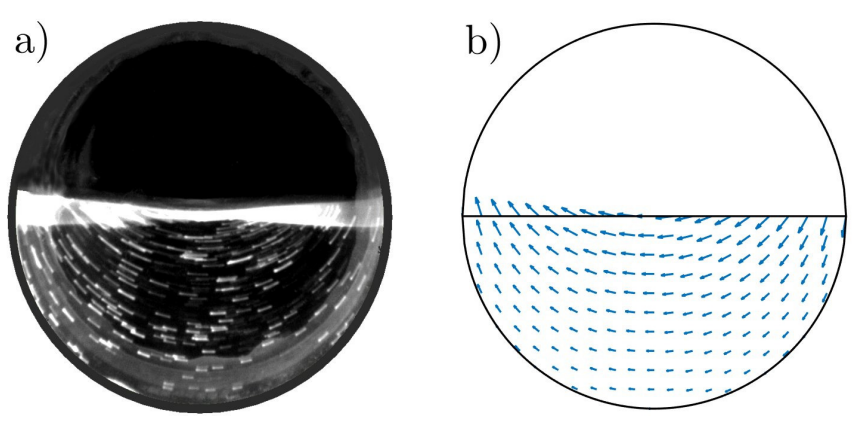

FIG. 5. a. Picture of the sloshing motion of water with a still cylinder (radius $R=5 \mathrm{~cm}$ ). Exposure time $=1 / 10 \mathrm{~s}$. The picture shows neutrally buoyant tracers (polystyrene spheres of density $1.06 \mathrm{~g} / \mathrm{cm}^{3}$ and diameter $\left.700 \mu \mathrm{m}\right)$. b. Velocity field computed from the potential flow given by Ibrahim ${ }^{21}$. Note that the velocity decreases with increasing distance from the free surface.

The potential from which the velocity is derived is given in terms of a $\operatorname{series}^{20,21}$ and the corresponding velocity field is plotted in figure $5 \mathrm{~b}$. A few comments can highlight the differences with a rigid-body rotation. First, the velocity clearly decays with increasing distance from the free surface whereas it increases linearly in the 
case of a rigid-body rotation. Second, one can notice that the streamlines are not half-circles centered on the center of the bottle. Instead, the streamlines are flatter near the free surface. Finally, the velocity at the center of the bottle is not zero. It is purely horizontal and oscillates back and forth. The theoretical velocity field is remarkably similar to the experimental observations. The picture in figure 5a shows neutrally buoyant markers indicating the motion of the fluid in a bottle pushed and held against a wall. This excellent agreement supports the assumption that the viscosity of the fluid plays no major role in the slosh dynamics, aside from the boundary layer discussed earlier.

\section{B. Experimental validation}

Experiments were performed by pushing a half-empty cylinder against a wall in order to induce fluid motion. The cylinder is then firmly held still against the wall and the slosh dynamics of the water is studied. The altitude of the free surface against the curved side of the cylinder was recorded (at a frame rate of 500 FPS) and measured through particle tracking (see inset in figure 6).

A simple way to check whether the potential flow is an accurate description of the actual flow is to measure the frequency of the oscillations. The Fourier transform of the signal is shown in figure 6 . The frequencies of the first three modes of sloshing can be analytically predicted $^{21}$ $\left(\omega_{1} \simeq 1.17 \sqrt{g / R}, \omega_{2} \simeq 2.17 \sqrt{g / R}\right.$ and $\left.\omega_{3} \simeq 2.82 \sqrt{g / R}\right)$ and are indicated by red lines.

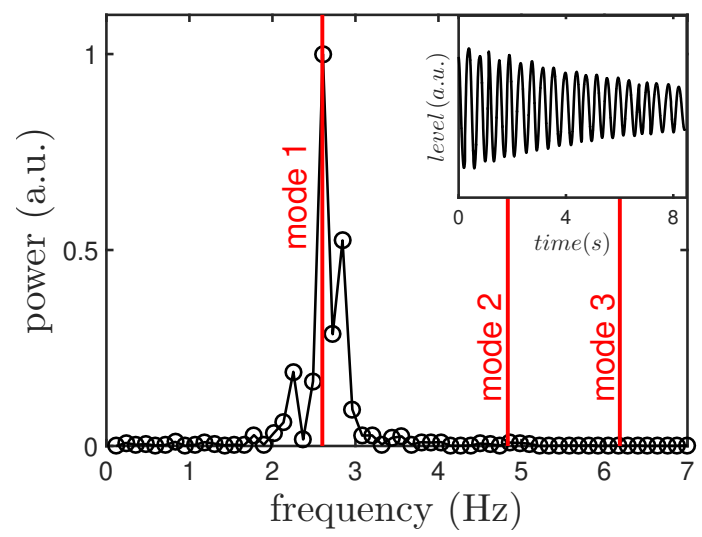

FIG. 6. Fourier power spectrum of the motion of the free surface in a bottle first pushed and then held still. The experimental maximum coincides with the theoretical frequency of the first sloshing mode. The inset shows the damping of the amplitude over long times.

A decay of the amplitude of oscillations is visible on the inset and is a long-term effect of the viscosity of the fluid. However, the agreement between the observed frequency and the prediction under the assumption of an ideal fluid is excellent, again supporting the hypothesis that the viscosity plays no major part in the flow (although it causes a slow damping). Moreover, even if the force initially applied to the bottle induces a sloshing motion which is more complex that an oscillating flat surface, the first sloshing mode is always dominant. The second or third modes could be observed if the cylinder were shaken at the corresponding resonance frequency, but in the case of an inherently asymmetrical initial push mode 1 always dominates. Therefore in the following only the first mode (in which the free surface remains flat although obviously not horizontal) will be considered.

\section{A SOLID BODY TOY MODEL}

Having understood that the rotation of the fluid and of the bottle are decoupled over short times (less than 10s) one can propose a mechanical model for the motion of a half-empty bottle in which the fluid is seen as a solid half-cylinder. This simplification is obviously inappropriate (as discussed in section III) but allows for a derivation and analysis of the equations of motion of a half-cylinder, representing the water, mounted on wheels, representing the bottle (see figure 7a). The mass and moment of inertia of the water are denoted $m$ and $I_{G}$ (computed around its center of mass, $G$ ) while those of the bottle alone are denoted $M$ and $J$. The horizontal position of the bottle is noted $x$, and the inclination of the half-cylinder $\theta$. The distance between the center of the bottle and the center of mass of the water is noted $l=O G=4 R / 3 \pi$. The model presented here is very similar to that of Jackson ${ }^{8}$ in which further technical details can be found. a)
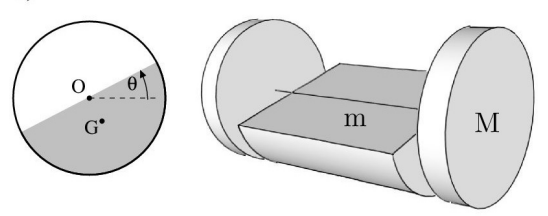

b)

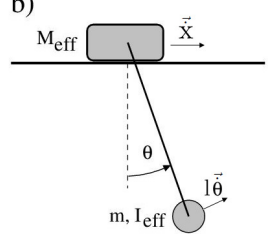

FIG. 7. a): Schematics of a half-cylinder mounted on wheels equivalent to a pendulum attached to a sliding block (b) with effective moment of inertia and masses.

Assuming a slip-free rotation of the bottle allows one to express its rotation speed: $\dot{x} / R$. If all sources of dissipation are neglected (air drag, solid and rolling friction, viscosity of the fluid etc) the equations of motion can be derived from the Euler-Lagrange equations using the kinetic energy $T$ and potential energy $V$ :

$$
\left\{\begin{array}{l}
T=\frac{1}{2} M \dot{x}^{2}+\frac{1}{2} J\left(\frac{\dot{x}}{R}\right)^{2}+\frac{1}{2} m(\overrightarrow{\dot{x}}+l \overrightarrow{\dot{\theta}})^{2}+\frac{1}{2} I_{G} \dot{\theta}^{2} \\
V=m g l(1-\cos \theta)
\end{array}\right.
$$

In the small-angle approximation, the equations of motion therefore read: 


$$
\left\{\begin{array}{l}
\left(M+\frac{J}{R^{2}}\right) \ddot{x}+m(\ddot{x}+l \ddot{\theta})=0, \\
\left(I_{G}+m l^{2}\right) \ddot{\theta}=-m g l \theta-m l \ddot{x} .
\end{array}\right.
$$

Interestingly these equations are equivalent to those of an analogue system consisting in a pendulum with an effective moment of inertia $I_{\text {eff }}=I_{G}+m l^{2}$ attached to a block sliding with no friction (see figure $7 \mathrm{~b}$ ) with an effective mass $M_{\text {eff }}=M+J / R^{2}$. Note that the effective moment of inertia, $I_{\text {eff }}$, is simply equal to the moment of inertia of the half-cylinder around the center of the bottle $O: I_{O}$. The equations of motion can be easily combined and solved ( $\operatorname{see}^{8}$ for details):

$$
\left\{\begin{array}{l}
\ddot{\theta}=A \sin (\Omega t+\varphi) \\
\ddot{x}=-A \frac{m}{m+M_{e f f}} l \sin (\Omega t+\varphi) \\
\text { where } \Omega^{-2}=\frac{l}{g}\left(\frac{I_{O}}{m l^{2}}-\frac{m}{M_{e f f}+m}\right) .
\end{array}\right.
$$

where $A$ (in rad.s ${ }^{-1}$ ) and $\varphi$ (in rad) are constants which depend on the initial conditions.

\section{SLOSH DYNAMICS IN A ROLLING BOTTLE}

\section{A. Adaptation of the theory to a rolling bottle}

The results presented above can be combined to study the motion of a half-empty bottle. The moment of inertia $I_{O}$ in equation 3 simply needs to be replaced by that of the first mode of sloshing given in section III: $I_{s}=$ $\pi \rho R^{4} L\left(\frac{4}{\pi^{2}}-\frac{1}{4}\right)$. Moreover, for simplicity, the moment of inertia of the bottle itself is assumed to be $J=M R^{2}$ and the effective mass defined in section IV is then simply $M_{\text {eff }}=2 M$. The angular frequency of the oscillations is therefore given by:

$$
\Omega^{-2}=\frac{l}{g}\left(\frac{I_{s}}{m l^{2}}-\frac{m}{2 M+m}\right)
$$

Note that the ratio $I_{s} / m l^{2}$ is only a geometrical constant $(\simeq 1.72)$ since $l=4 R / 3 \pi$. Moreover, the right-hand term is ensured to be positive since the term in parenthesis can be rewritten as: $I_{G} / m l^{2}+2 M /(2 M+m)$.

Knowing the initial conditions therefore allows one to determine the constants $A$ and $\varphi$ in equation 3 and to integrate them. The following paragraphs will discuss the motion in two different setups: a half-empty bottle bouncing on a wall and a half-empty bottle rolling down a steep slope.

\section{B. Soft collision of a half-empty bottle}

\section{Initial conditions}

As explained above, the motion strongly depends on initial conditions. When a bottle is manually pushed, it is difficult to properly determine what force (i.e. acceleration) or velocity is imposed and the corresponding initial conditions remain unclear. In order to study a well-defined set of initial conditions, a half-empty bottle rolling on a flat surface without any oscillations is sent to bounce on a wall (figure 8). The initial steady velocity, $v_{0}$, is achieved by letting the bottle slowly accelerate on a gentle slope (less than $1^{\circ}$ ). The wall is made of soft wood in order to minimize the energy dissipation. Although the collision must be somewhat inelastic we found that an empty bottle loses less than $5 \%$ of its speed after a collision against the wall. Moreover, very little energy is dissipated in the fluid boundary layer. Therefore, all sources of dissipation during the collision are neglected.

When the bottle hits the wall (and while it remains in contact with the wall), the water rises as the initial kinetic energy is converted into potential gravitational energy. The surface then recoils back to horizontal at which time $(t=0)$ the bottle leaves the wall and rolls forward while the fluid keeps oscillating. However, an empty bottle tends to almost instantly bounce off the wall. There is therefore a competition between the relatively slow rise of the water (which tends to pull the system against the wall) and the rapid rebound of the bottle itself (which tends to pull the system away from the wall). If the mass of the water dominates, only the first mode of sloshing is triggered and a set of initial conditions can be determined. In this case, the initial kinetic energy of the bottle itself prior to the collision not only does not contribute to the rise of the water but instead must be subtracted. This procedure is a reproducible and controlled way to set initial conditions: $x_{0}=0, \dot{x}_{0}=0$, $\theta_{0}=0$ and where $\dot{\theta}_{0}$ is given by

$$
\frac{1}{2} I_{s} \dot{\theta}_{0}^{2}=\frac{1}{2} m v_{0}^{2}-\frac{1}{2} M v_{0}^{2} .
$$

\section{Analytical solution}

Under these conditions the motion of the fluid and of the bottle is given by the solutions of equation 3 :

$$
\left\{\begin{array}{l}
\dot{\theta}=\dot{\theta}_{0} \cos \Omega t \\
\dot{x}=\beta v_{0} \frac{(m-M)^{1 / 2}}{2 M+m}(1-\cos \Omega t),
\end{array}\right.
$$

where $\beta=\frac{8}{3 \sqrt{2}} \frac{1}{\sqrt{16-\pi^{2}}} \simeq 0.76$.

Note that neglecting the mass of the end-caps, the ratio of masses can be expressed in terms of thickness, $e$, and density (relative to that of water), $d$, of the bottle itself: $M / m=4 e / R d$. 


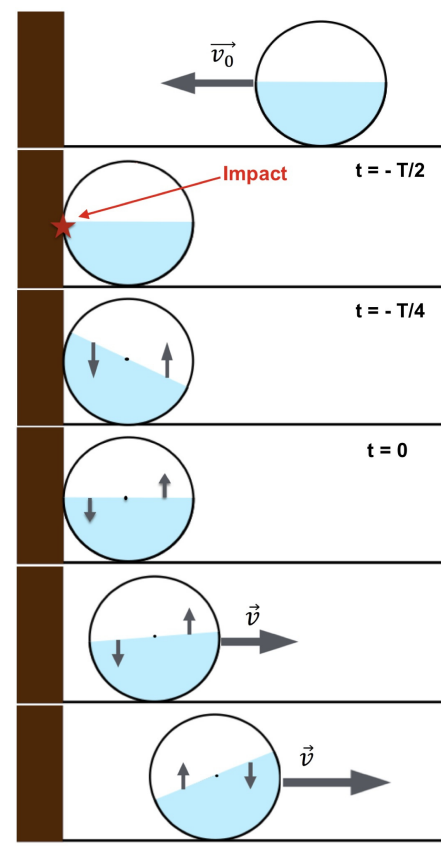

FIG. 8. A half-empty bottle is sent to bounce on a wall. During the impact the fluid is set into rotation which triggers oscillations in the velocity when the bottle leaves the wall.

\section{Experimental validation}

A cylindrical bottle of radius $R=50 \mathrm{~mm}$ and length $L=70 \mathrm{~cm}$, made of Plexiglas, was used to validate the theoretical predictions. The mass of the empty bottle is $M=0.910 \mathrm{~kg}$ and the mass of water used (corresponding to a half-empty bottle) is $m=2.780 \mathrm{~kg}$. The motion of the bottle was recorded at 30 FPS and the position was measured using particle tracking and the velocity was com-

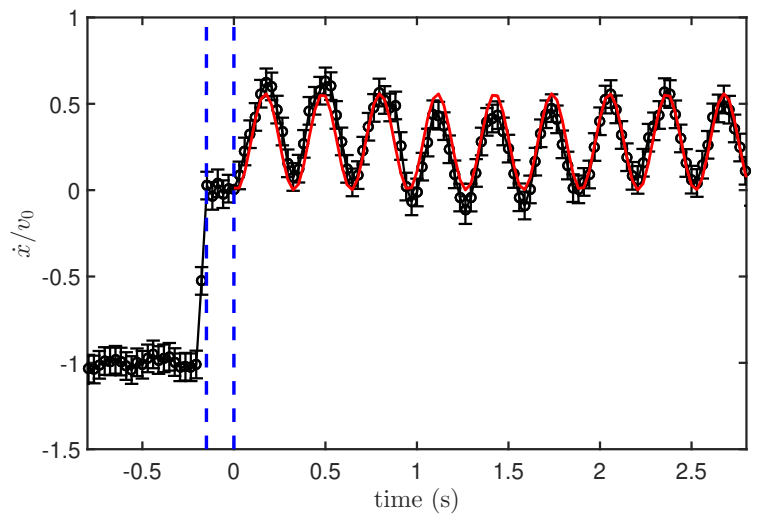

FIG. 9. Velocity of the half-empty bottle experiencing a soft collision against a wall. The velocity is constant before the impact (first dashed line) and oscillations are triggered after the bottle leaves the wall (second dashed line). The red line shows the velocity predicted using equation 6 . There are no fitting parameters and the agreement is excellent. puted as its numerical derivative. The results are presented in figure 9 . The velocity prior to the impact, $v_{0}$, is rather constant (with small fluctuations, in part due to the image processing) while after the impact (indicated by the two dashed lines) the velocity largely oscillates. One can see that the bottle undergoes a stop-and-go motion since the velocity periodically reaches zero. This feature is visible on the space-time diagram in figure 1 .

This motion is also predicted by our theory (red line). It is worth noting that there are no fitting parameters. The initial velocity is measured as the average before the impact, and the amplitudes, frequency and phase are those predicted by equation 6 . The duration of the impact itself is given by a half-period of the oscillations. The agreement between the experimental data and the theory is excellent, supporting the validity of the numerous hypotheses made (first mode of sloshing, small angles, energy conservation and inviscid fluid). Knowing the time of impact, one can therefore entirely determine the motion of the bottle after the bounce.

\section{Half-empty bottle on a steep slope}

\section{Analytical solution}

Another protocol used to produce reproducible and controlled initial conditions consists in letting a halfempty bottle roll down an incline plane of slope $\alpha$, which must remain small enough to ensure a slip-free rolling motion of the bottle. The inclination of the free surface, $\theta$, is measured in the frame of reference on the plane. The gravitational potential energy of the bottle and the fluid needs to be included in the Euler-Lagrange equations which yield (in the small-angle approximation):

$$
\left\{\begin{array}{l}
2 M \ddot{x}+m(\ddot{x}+l \ddot{\theta})=(M+m) g \alpha, \\
I_{s} \ddot{\theta}=-m g l(\theta-\alpha)-m l \ddot{x} .
\end{array}\right.
$$

For a bottle initially $(t=0)$ at rest:

$$
\dot{x}=\frac{M+m}{2 M+m} g \alpha t+\frac{m(M+m)}{(2 M+m)^{2}} \alpha l \Omega \sin \Omega t
$$

The velocity is composed of two separate terms: the first one is the average constant acceleration (smaller than $\alpha g$ as discussed in section II), while the second indicates the velocity fluctuations.

It may seem counter-intuitive that releasing the bottle initially at rest should trigger oscillations, but it can be understood with a hand-waving argument. In the moving non-inertial frame of reference of the bottle an inertial force applies to the water, shifting the equilibrium position to $\theta_{e q}=\alpha M /(M+m)$. The amplitude of the oscillations (of the inclination of the free surface) is therefore given by $\theta_{0}-\theta_{e q}=\alpha m /(M+m)$. 


\section{Experimental validation}

A cylindrical bottle of radius $R=44 \mathrm{~mm}$ and length $L=170 \mathrm{~mm}$, made of Plexiglas, was used to validate the theoretical predictions. The mass of the empty bottle is $M=0.140 \mathrm{~kg}$ and the mass of water used (corresponding to a half-empty bottle) is $m=0.510 \mathrm{~kg}$. The motion of the bottle on a table inclined at an angle of $\alpha=15^{\circ}$ was recorded at 500 FPS and the position was measured using particle tracking and the velocity was computed as its numerical derivative. The results are presented in figure 10 .

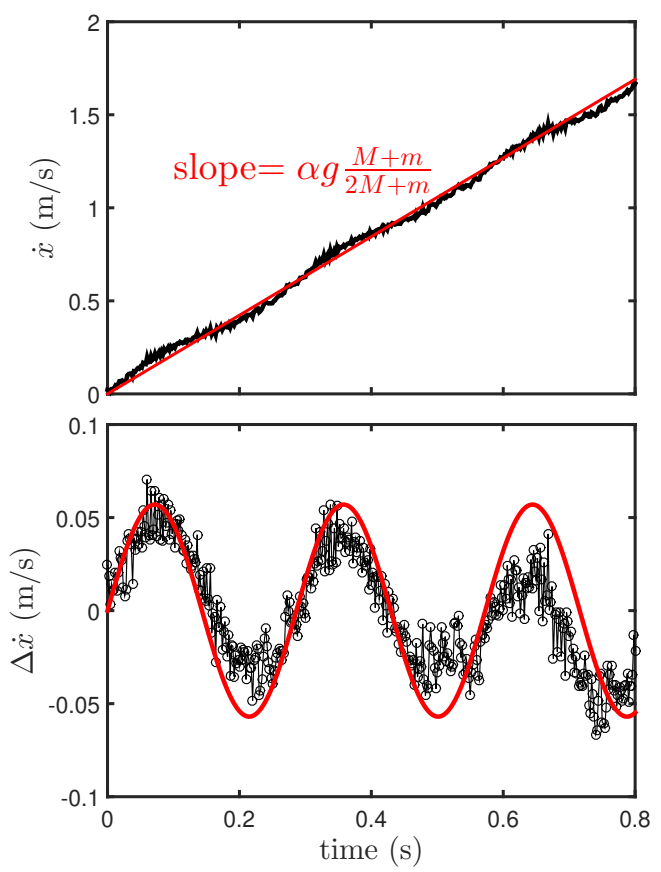

FIG. 10. Top: velocity of a bottle released on a $15^{\circ}$ slope. On average the bottle undergoes a constant acceleration but oscillations in the velocity are visible. The red line shows the predicted average acceleration. Bottom: deviation from the constant acceleration highlighting the oscillations: experimental data (black) and theoretical prediction (red).

The experimental data confirms the theoretical predictions: the bottle rolls down the slope with an average constant acceleration and oscillations in speed are clearly visible. The experimental acceleration is in excellent agreement with the predicted value. However, for longer times a discrepancy appears and might be attributed to the air drag acting on the bottle or to the failure of the decoupling assumption. The velocity fluctuations, $\Delta \dot{x}$ defined as the difference in velocity from the linear behavior, are shown in the bottom of Figure 10 where the red line shows the theoretical predictions. It should be emphasized once again that there are no fitting parameters: all values are deduced from the simple measurement of the masses and sizes. The amplitude, frequency and phase are those given by equation 8 . The fluctuations are small (typically $5 \%$ of the speed) and very sensitive to the imperfections of either the table or the bottle. Still, the experimental data shows a good agreement with the theory. One can see that the amplitude decays but the initial magnitude of the first few oscillations is well captured while the initial phase and the frequency are very well predicted. Again, the agreement between these experimental results and the theory validates the seemingly strong assumptions made.

\section{CONCLUSION}

In conclusion we were able to successfully model the motion of a half-empty bottle rolling on a flat surface. We showed that over the duration of a typical experiment the rotation of the water and of the bottle are decoupled, which can also be predicted from the NavierStokes equation. A simple mechanical toy-model allowed for the derivation of the equation of motion while the exact motion of the fluid is well described by the first mode of sloshing under the assumption of an inviscid fluid. Experiments performed on a bottle bouncing on a wall and on a steep slope provided experimental validation of the predicting power of the theoretical predictions.

The limitations of our work could deserve further attention. It is clear that the decoupling of the rotation of the fluid and the bottle should not hold for smaller radii: if a test-tube of radius $R=1 \mathrm{~cm}$ is used, the diffusion time is of the order of a few seconds and the whole fluid can be set into a rigid-body rotation. The same conclusion can be drawn if a more viscous fluid is used: vegetable oils are typically 50 times more viscous than water, leading to a diffusion time of the order of one second. Finally we should recall that the equations were derived in the small-angle approximation and that an exact study of larger amplitudes might reveal surprising results.

The potential flow gives an accurate description of the motion of the water in the bottle but the effect of the viscosity is visible in figure 6 . A comprehensive study of the viscous dissipation in the boundary layer might deserve further attention. Similarly, the effect of static and rolling friction of a bottle rolling on a surface, as well as that of drag caused by the surrounding air, could be studied to provide one with a more realistic description of the motion over long times.

It would be interesting to vary the filling fraction of the bottle. Obviously, there can be no oscillations in a bottle either completely full or empty. Therefore, there must exist an optimal filling fraction for the oscillatory motion studied in this paper. It is unclear whether the optimal value should correspond to a filling fraction of one half. 


\section{ACKNOWLEDGMENTS}

The authors acknowledge support from the PALSE program of the University of Lyon Saint-Etienne, the University Lyon Claude Bernard, the Société Française de Physique and from the École Normale Supérieure de Lyon and its Physics Department and Laboratoire de Physique. We are thankful to B. Capitaine for techni- cal help and A. Chemin, A. Guittonneau, C. Gouiller, C. Malciu and J. Sautel for fruitful discussions. The work presented here was done in preparation for the International Physicists Tournament (http://iptnet.info), a world-wide competition for undergraduate students. The authors are grateful to both local and international organizing committees of the International Physicists Tournament for having put together an exciting event.
* nicolas.taberlet@ens-lyon.fr

1 J. V. Wehausen and E. V. Laitone, Surface waves (Springer, 1960).

2 A. M. Davis and P. D. Weidman, Physics of Fluids (1994present) 12, 971 (2000).

3 D. Henderson and J. Miles, Journal of Fluid Mechanics 275, 285 (1994).

${ }^{4}$ H. F. BAUER, AIAA Journal 1, 2601 (1963).

${ }^{5}$ F. T. Dodge et al., The new" dynamic behavior of liquids in moving containers" (Southwest Research Inst. San Antonio, TX, 2000).

${ }^{6}$ P. Pal, International Journal of Recent Trends in Engineering 1 (2009).

7 M. Eswaran and U. K. Saha, Ocean Systems Engineering 1, 131 (2011).

8 K. Jackson, J. Finck, C. Bednarski, and L. Clifford, American Journal of Physics 64, 277 (1996).

9 S. Lin, N. Hu, T. Yao, C. Chu, S. Babb, J. Cohen, G. Sangiovanni, S. Watt, D. Weisman, J. Klep, et al., The Physics Teacher 53, 548 (2015).
10 G. Ireson and J. Twidle, International Journal of Mathematical Education in Science and Technology 36, 423 (2005).

11 S. J. Micklavzina, Physics education 39, 38 (2004).

12 M. Bunge, American Journal of Physics 25, 211 (1957).

13 D. Zaslavsky and D. Kirkham, American Journal of Physics 33, 677 (1965).

14 T. Kambe, Elementary Fluid Mechanics (2007).

15 O. N. Mikhailov M.D., Unified Analysis and Solutions of Heat and Mass Diffusion (1994).

16 J. W. MacDougall, American Journal of Physics 44 (1976).

17 N. P. Singh, S. C. Gupta, and B. R. Sood, American Journal of Physics 70 (2002).

18 H. D. Wiederick and N. Gauthier, American Journal of Physics 51 (1983).

19 J. Rayleigh, The theory of sound, 1st ed. (London, Macmillan and co., 1887).

20 H. Lamb, Hydrodynamics, 3rd ed. (University Press, 1916).

21 R. Ibrahim, Liquid sloshing dynamics: theory and applications, 1st ed. (Cambridge University Press, 2005). 